\title{
Correspondence
}

\section{The scope of rheumatology}

\author{
Open letter to Dr Malcolm Jason
}

Dear Malcolm,

It's not often that I write you an open letter but your Viewpoint ${ }^{1}$ has exercised me somewhat over the last few weeks. It has achieved its function of being provocative: just why do I, a rheumatologist, call myself a consultant in rehabilitation medicine, and, if it is a nonspecialty, why do I find myself so usefully occupied? Perhaps I shouldn't be doing it. After all, as you so rightly say, the breadth of the rheumatologist's field is great and it is not easy to keep pace with all the developments within it-and not a few of our number are super-super specialists covering only a part of the field. Rheumatologists are exemplary fellows - they do practice the total management of their patients whereas (dare I say it) some neurologists seem to feel their brief is complete when an exotic label is attached to their patient. Some temperaments seem to cope better than others with patients whose disease is never cured (how many are?) and with patients who may return to one's clinic for a quarter of a century. Rheumatologists again seem well suited to such work, and this is probably at least partly why they applied their expertise to other groups of patients in need of it and entered and gained eminence in the field of rehabilitation, so that about half of those rheumatologists and senior registrars in rheumatology have had training in rehabilitation. You rightly say that such work should not be confined to rheumatologists, but few other fields provide training in rehabilitation for their junior grades, so how are they to be at least made aware of their responsibilities? As I see it, it needs practitioners of rehabilitation to come from rheumatology at least initially to train all comers. They need to be of high academic status - to combat the view that rehabilitation is a 'soft specialty'; they may need to retain their specialist base to give them credence. Here in Leeds we train medical students at all clinical levels, rotating SHOs, health visitors, social workers, dietitians, everyone it seems, including embryo hospital administration. I believe it is beginning to make a differenceafter 5 years.

There is a supposition which worries me. On the one hand there is the cry to link rheumatology with general medicine, and on the other a subtle denigration of rehabilitation as an also-ran. Now our rehabilitation unit deals mostly with heavy disability: one patient has multiple sclerosis with above-knee amputations, another paraplegia due to $B_{12}$ deficiency, with bad social conditions, and yet another schizophrenia and hypertension and hemiplegia. Multiple handicaps are, you might say, the norm in our unit. A marvellous medley of medicine abounds-dermatomyositis, Wegener's granulomatosis, a Blalock's operation done by Sir Russell Brock in the early $1950 \mathrm{~s}$ - all have been our patients over the last few months. Not enough general medicine?

Why is the rehabilitation part of a post regarded as a chore? There is a need, certainly in every medical school, for someone to teach and undertake research in the subject. There is a need (at regional level or below) for someone to deal with multiple and severe disability. But rehabilitation patients cannot be too far from home, and the doctor cannot be too far removed from the population he will care and plan for. He needs to work closely with social services to ensure that facilities for the disabled aged 16-64 become adequate. (I know of no area now which provides sufficient sheltered work, sheltered housing, purpose-built housing, day hospital and care facilities and young-disabled units). There are few areas where any one adult physician takes a particular and continuing medical interest in the over 16-year-old with physical handicap in any way comparable to the care paediatrics provide. There is usually no medical focus of attention. Where one exists-I believe I do, in Leeds-one finds that the development of clinical expertise, teaching, research, and health care planning for the relevant patients provides a stimulating job not unworthy of any rheumatologist, however academic. It is time to take stock and realise that within the ranks of rheumatologists are those who would enjoy the creativity of carving out new posts responsive to their own expertise and aims: they are unlikely to run short of work before retirement.

\section{AnNe Chamberlain Consultant Physician in Rehabilitation Medicine, The General Infirmary at Leeds}

\author{
Reference \\ 1 Jayson M I V. The scope of rheumatology. Ann Rheum \\ Dis 1980; 39: 528.
}

\section{Reply from Professor Jayson}

\section{Dear Anne,}

In my Viewpoint paper I emphasised the breadth of the field of rheumatology and the need to consider the management of degenerative disease, soft tissue rheumatism, and the physical reablement of the disabled arthritic as integral parts of the specialty and not requiring separate designation as 'rehabilitation.' Equally we believe that other specialties should assume similar responsibilities. There is a gradual change in the attitudes of society and the medical profession towards the disabled, and we are now seeing the training programmes of many specilties appreciating the need for total patient care. I think that the specialist in rehabilitation should be based in a centre of excellence such as your own, taking the very difficult and multiply handicapped problems and 
advising other departments on the development of their services and how to manage individual problems. The subtle denigration of which you speak is a consequence of the present structure in which rheumatologists are often asked to take over the care of another specialist's patients when they feel they can do no more. The process of rehabilitation should start from the time the patient is first seen and requires an attitude of mind by all members of the clinical team.

You know that I am an active supporter of the development of the specialty of rehabilitation. I believe that the best way forward is the emergence of the specialty of rehabilitation in its own right rather than as an appendage to rheur:atology.

Malcolm I. V. JAYSON Professor of Rheumatology, Rheumatic Diseases Centre, University of Manchester

\section{Reactive arthritis associated with campylobacter enteritis}

Sir, It was with interest that we read of the recent survey by Gumpel et al. ${ }^{1}$ of patients in the Harrow Health District infected with campylobacter species during 1978. We are currently analysing the results of a similar survey of a single milk borne outbreak of Campylobacter jejuni originating from one dairy in a rural district south of Aberdeen. ${ }^{2}$

One hundred and twenty-six patients under the care of 2 general practitioners, who attend all patients in the relevant area, were infected with Campylobacter jejuni in the months of January and February of 1979 and were traced through the records of the Regional Laboratory, City Hospital, Aberdeen, where all samples were sent. One hundred and thirteen patients continue to reside in the area and their general practice records have been examined. Two patients have subsequently attended hospital clinics. One of these, a male aged 41 , presented within 1 month of his gastroenteritis with left-sided sciatica to a local orthopaedic colleague. Six months previously he had had a right-sided sciatica. His notes indicate that he had evidence of nerve root irritation, and his symptoms spontaneously resolved. The second patient was an 8-year-old girl who presented 2 months after initial infection with intermittent fever, muscle aching, and joint pains. She continues to have episodes of diarrhoea. She was seen by a paediatric colleague, who could find no clinical abnormality of the joints. Her ESR was normal. There was no evidence from repeated stool cultures of reinfection within the family.

A third patient, a 34-year-old male, did not attend hospital, but presented to his general practitioner about 1 month after infection with a right olecranon bursitis. No other patients' records indicate any medical attention for musculoskeletal complaints within 3 months of their bowel infection.

To complete the survey symptomatic patients are to be contacted and their clinical history reviewed, personally, On the basis of the results of this survey so far the frequency of inflammatory joint disease would appear to be considerably less in this single outbreak of Campylobacter jejuni enteritis in the community than in the 33 hospitalised patients seen by Gumpel et al. It is of interest that they were unable to identify any examples of arthritis in patients who had not been hospitalised for their infection. This, together with the results from our own survey, suggests that the severity of the gastroenteritis may be of importance in addition to genetic factors in determining the development of a subsequent reactive arthritis. Alternatively, the sample from our study may be less biased than the Harrow series. Our study is of a single outbreak during which close cooperation between the Regional Laboratory and the general practitioners ensured the collection of faecal samples from all symptomatic patients presenting together with contacts, thus ensuring as complete a sample as possible. It is well recognised that hospitalised patients are not representative of a population sample with the same disease.

C. J. EASTMOND*
T. M. S. REID
J. A. N. RENNIE*
${ }^{*}$ Department of Rheumatology* and
$\dagger$ Regional Laboratory, City Hospital,
Urquhart Road, Aberdeen AB9 $8 A U$.

\section{References}

1 Gumpel J M, Martin C, Sanderson P J. Reactive arthritis associated with campylobacter enteritis. Ann Rheum Dis 1981; 40: 64-5.

2 Porter I A, Reid T M S. A milk-borne outbreak of campylobacter infection. J Hyg 1980; 84: 415-9.

\section{CRVO and scleroderma}

Sir, In their interesting article Littlejohn et al..$^{1}$ described a case of central retinal vein occlusion (CRVO) in a patient with progressive systemic sclerosis (PSS). According to the authors this might have been a manifestation of sclerodermatous vascular disease at the level of the fundus oculi. While this hypothesis is doubtless correct, we would like to point out that occlusion of the central retinal vein is rare in PSS patients and, in general, in patients with connective tissue disorders. By means of simple ophthalmoscopy we failed to find this lesion in a nonselected series of 71 patients with PSS. Grade III or IV hypertensive retinopathy was present in 2 of these patients with sclerodermatous malignant hypertension. We found abnormalities of the fundus vessels in another 24 , but 10 of these patients were above 60 years of age. Other eye lesions found in the above series of 71 patients were: mild choroiditis (2), nodular episcleritis (1), mild keratitis (2), markedly reduced tear secretion (4), and induration of the lower lid (48). CRVO was found only in 2 out of 31 cases of undefined connective tissue disease (one of the 2 with probable polyarteritis nodosa). On the other hand CRVO was not found in patients with systemic lupus erythematosus (31 cases), dermatomyositis and polymyositis (13 cases), undifferentiated 